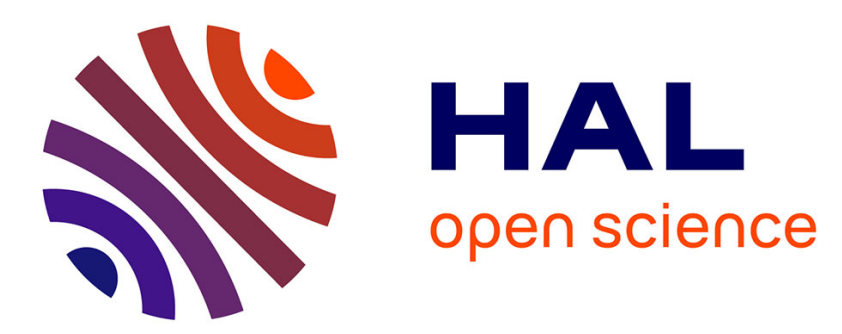

\title{
Extreme optical fluctuations in lumped Raman fibre amplifiers
}

\author{
Kamal Hammani, Christophe Finot
}

\section{To cite this version:}

Kamal Hammani, Christophe Finot. Extreme optical fluctuations in lumped Raman fibre amplifiers. Journal of Optics, 2013, 15 (6), pp.064009. 10.1088/2040-8978/15/6/064009 . hal-00815524

\section{HAL Id: hal-00815524 \\ https://hal.science/hal-00815524}

Submitted on 18 Apr 2013

HAL is a multi-disciplinary open access archive for the deposit and dissemination of scientific research documents, whether they are published or not. The documents may come from teaching and research institutions in France or abroad, or from public or private research centers.
L'archive ouverte pluridisciplinaire HAL, est destinée au dépôt et à la diffusion de documents scientifiques de niveau recherche, publiés ou non, émanant des établissements d'enseignement et de recherche français ou étrangers, des laboratoires publics ou privés. 


\title{
Extreme optical fluctuations in lumped Raman fibre amplifiers
}

\author{
K Hammani ${ }^{1,2}$ and C Finot ${ }^{1}$ \\ ${ }^{1}$ Laboratoire Interdisciplinaire Carnot de Bourgogne, UMR 6303 CNRS - Université de \\ Bourgogne, 9 avenue Alain Savary, BP 47870, 21078 Dijon Cedex, France \\ ${ }^{2}$ Now at Optoelectronics Research Centre, University of Southampton, Highfield, \\ Southampton, SO17 1BJ, United Kingdom \\ E-mail : christophe.finot@u-bourgogne.fr
}

\begin{abstract}
A numerical study about the generation of extreme events in lumped Raman fibre amplifier is performed. Evolutions of continuous or pulsed signals are analysed using crosscorrelations, spectra and probability density functions. For pulsed signals, the phase evolution is also explored. Both signal and Stokes cascaded waves are considered.
\end{abstract}

Keywords. Rogue Wave, statistical optics, optical fibre amplifier.

\section{Introduction}

The study of extreme statistics in nonlinear fibre optics is a topic that has recently stimulated much attention. At the end of the year 2007, an article has reported the experimental observation of significant fluctuations in the temporal domain [1]: by spectrally filtering the most red-shifted part of a supercontinuum spectrum generated in a photonic crystal fibre, Solli et al have analysed the statistical fluctuations of the corresponding temporal intensity and found a highly-skewed distribution that strongly differs from the Gaussian shape. By analogy with the typical L-shaped distribution observed for extreme events, the intense and extremely rare outliers of the distribution were named "optical rogue waves". Since then, the subject has become the focus of intense international research in the optical community as evidenced by the large number of studies published in the last five years $[2,3]$.

The initial works have focused on the passive propagation of picosecond pulses in photonic crystal fibres and those different studies carried out in the anomalous dispersion regime have highlighted the specific role played by Raman frequency shifted solitons that are also affected by third-order dispersion and resulting pulse-to-pulse interactions [4-6]. It has also theoretically and experimentally highlighted the importance of the initial stage of modulation instability and confirmed the crucial role of the nonlinear coherent structures such as fundamental solitons [7, 8], breathers [9, 10] and rational solitons such as the Peregrine soliton [11, 12]. 
However, observation of statistics that strongly deviate from a Gaussian distribution is absolutely not restricted to supercontinuum generation and can also be observed in telecommunication applications in the context of transmissions [13-15], Raman fibre lasers [16-18] as well as discrete amplification [1922]. More precisely, in this latest field, it has been shown that under certain conditions, statistical distribution of an amplified signal can be strongly reshaped during the amplification process. Examples of this deleterious degradation have been discussed for parametric amplifiers [19, 23, 24] and for Raman amplifiers [20, 25-28] : in both cases, a quasi-instantaneous gain and a low walk-off between the signal and a partially incoherent pump lead to a dramatic transfer of fluctuations from the pump to the signal in a co-propagating configuration.

In our previous works, we have numerically and analytically described the evolution of a continuous signal in a co-propagating amplifier in the presence of a low walk-off and pump depletion [26]. Using a series of temporal and spectral characterizations, we have experimentally confirmed the drastic consequences of the transfer of intensity fluctuations from the pump to a pulsed signal in a lumped Raman amplifier [27]. We have also shown that an adequate frequency shifted optical bandpass filter can isolate the most extreme structures [25].

In the present contribution, we rely on a set of numerical simulations to further illustrate the various conclusions we have been analytically, numerically or experimentally drawn in these previous works. We consider both continuous and pulsed signals and using correlations, spectra, probability density functions or eyes diagrams, we stress several important features of the resulting extreme fluctuations. We also discuss the properties of the Stokes cascaded wave that may develop during the amplification process as well as the phase of a high repetition rate pulsed signal.

Our paper will be thus organized as follows. We will first present the model and the parameters of the configuration we numerically study. Then, we will more specifically investigate the amplification of a continuous wave in the Raman lumped amplifier. Numerical simulations will be used to provide an accurate and close to complete picture of the temporal and spectral nonlinear dynamics. In the final part of the paper, we discuss the effects of the amplification on the phase and intensity profiles of a $40 \mathrm{Gbit} / \mathrm{s}$ signal.

\section{Numerical model and parameters under investigation}

During its evolution in a single mode optical fibre, the slowly-varying envelope of the electrical field $\psi(z, T)$ of an optical wave can be described by the extended nonlinear Schrödinger equation [29] :

$$
i \frac{\partial \psi}{\partial z}=-i \frac{\alpha}{2} \psi-\sum_{k=2.4}(i)^{k} \frac{\beta_{k}}{k !} \frac{\partial^{k} \psi}{\partial T^{k}}-\gamma \psi \int_{0}^{\infty} R\left(t^{\prime}\right)\left|\psi\left(z, t-t^{\prime}\right)\right|^{2} d t^{\prime}
$$

with $\beta_{k}$ describing the chromatic dispersive properties of the optical fibre at the signal wavelength (in our configuration $1550 \mathrm{~nm}), \gamma$ the Kerr nonlinear coefficient and $\alpha$ the optical losses. $R(t)$ is the silica response and includes both the instantaneous and the delayed Raman response. The Raman amplifier under investigation is based on a 500-metre highly nonlinear polarization-maintaining fibre (HNLF), with $\beta_{2}$ of $0.63 \times 10^{-3} \mathrm{ps}^{2} \cdot \mathrm{m}^{-1}, \beta_{3}$ of $6.83 \times 10^{-6} \mathrm{ps}^{3} \cdot \mathrm{m}^{-1}, \beta_{4}$ of $2.66 \times 10^{-7} \mathrm{ps}^{4} \cdot \mathrm{m}^{-1}$ and a nonlinearity $\gamma$ of 10 $\mathrm{W}^{-1} \cdot \mathrm{km}^{-1} \cdot z$ and $T$ are the propagation distance and the temporal coordinate in a reference frame moving at the group velocity of the wave. $\psi(0, T)$ is the initial field made of the temporal superposition of a linearly polarized pump wave $\psi_{P}$ and a co-polarized signal $\psi_{S}$ which is shifted by $\Omega=13.2 \mathrm{THz}$ with respect to the pump wavelength and therefore leads to the maximum gain response : $\psi(0, T)=\psi_{P}(0, T) \exp (+i \Omega T)$ $+\psi_{S}(0, T)$. In order to investigate the transfer of energy to a cascaded Stokes wave and contrary to [25, 
26], we do not reduce Eq. (1) to a set of two coupled nonlinear Schrödinger equations. Compared to the widely used models for Raman amplification which rely on a power analysis [30-32], the model we implement here is based on the evolution of the scalar electrical field, which enables us to describe the dynamics, both in the temporal and spectral domains and to discuss the evolution of the phase of the pulse. Even if a rigorous modelling of the Raman amplification process in a non polarization-maintaining fibre would require the use of a full-Manakov PMD equations [31], the comparison between numerics and experiments made in our previous works [25, 26] have shown that model based on Eq. (1) could still provide many insights for a qualitative understanding of the appearance of giant spikes when the polarization of the pump beam is scrambled.

The crucial point here is that the pump used in our study is a partially incoherent pump $\psi_{P}$ that we modelled by a random wave whose Fourier transform is Gaussian-shaped with $\delta$-correlated random spectral phases $\varphi(\omega)$ uniformly distributed between $-\pi$ and $\pi$ [33-35] :

$$
\tilde{\psi}_{P}(\omega, 0) \propto \exp \left(-2 \ln (2) \frac{\omega^{2}}{\Omega_{L}^{2}}\right) \exp (i \varphi(\omega))
$$

$\Omega_{L}$ is the spectral full-width at half maximum (FWHM) of the pump, $\Omega_{L}=17.7 \mathrm{GHz}$, which corresponds in the temporal domain to intensity fluctuations having a minimum temporal FWHM of $25 \mathrm{ps}$. We will consider in our numerical study an average pump power of $0.5 \mathrm{~W}$, leading to an average gain of $11.2 \mathrm{~dB}$. Note that, because the spectrum $\left|\tilde{\psi}_{P 0}(\omega)\right|^{2}$ is $\delta$-correlated, the wave exhibits fluctuations that are statistically stationary in time [35]. Considering the standard probability density function (pdf) transformation rules (we refer the reader to Ref. [35] for details), one obtains the following expression of the pdf of the pump power $P_{\mathrm{P} 0}=\left|\psi_{P}(0, T)\right|^{2}$ which is well-known for a polarized thermal source [24, 35]:

$$
p d f\left(P_{P 0}\right)=\frac{1}{\left\langle P_{P 0}\right\rangle} \exp \left(-\frac{P_{P 0}}{\left\langle P_{P 0}\right\rangle}\right)
$$

Compared to previous studies dealing with fluctuations of a few tens of percent of the peak-power of pulses delivered by nanosecond Q-switched laser pumps [21, 32, 36-38], such a partially incoherent pump shows much larger deviations.

Regarding the properties of the initial signals, two kinds of signals will be numerically investigated. First, we will study the evolution of a purely continuous wave, i.e. a monochromatic wave with an average power of $0.5 \mathrm{~mW}$. Then, we will focus on the evolution of a Gaussian pulse train having a $6.25 \mathrm{ps}$ FWHM and an initial peak-power of $5 \mathrm{~mW}$.

\section{Analysis of the evolution of a continuous seed}

We first investigate the evolution of a continuous signal. We have outlined in previous works $[20,26]$ that a continuous seed evolving in a Raman fibre amplifier with low walk-off between pump and signal experiences a severe transfer of intensity noise from the partially incoherent pump to the signal leading to the generation of rare and intense spikes and significant spectral distortions. Figure (1) illustrates the emergence of such abnormally high intensity events: the intensity profile of the initial pump is plotted on a 4 ns range (Figure 1(a), subplot 1) and is compared with the resulting intensity profiles at the signal and Stokes wavelengths (Figure 1(b-c)). One can clearly make out that the amplified signal strongly differs from the initial continuous seed by exhibiting giant spikes. Events with an intensity being four hundred times and three thousand times larger than the average powers are recorded in the signal and cascaded wave respectively. A magnified view of the largest peak (subplots 2) confirms the tight connection 
between the pump fluctuation and the signal and Stokes cascade waves: they are close to being synchronized and of similar temporal duration. Physically, those extreme events essentially originate from the nonlinear transfer of pump to Stokes relative intensity noise and they do not result from a spontaneous process deriving from quantum noise $[31,39,40]$. Consequently and as discussed in $[25,26]$, most of the physics underlying the nonlinear temporal and spectral dynamics can be qualitatively caught by considering a simple transform-limited Gaussian pulse.

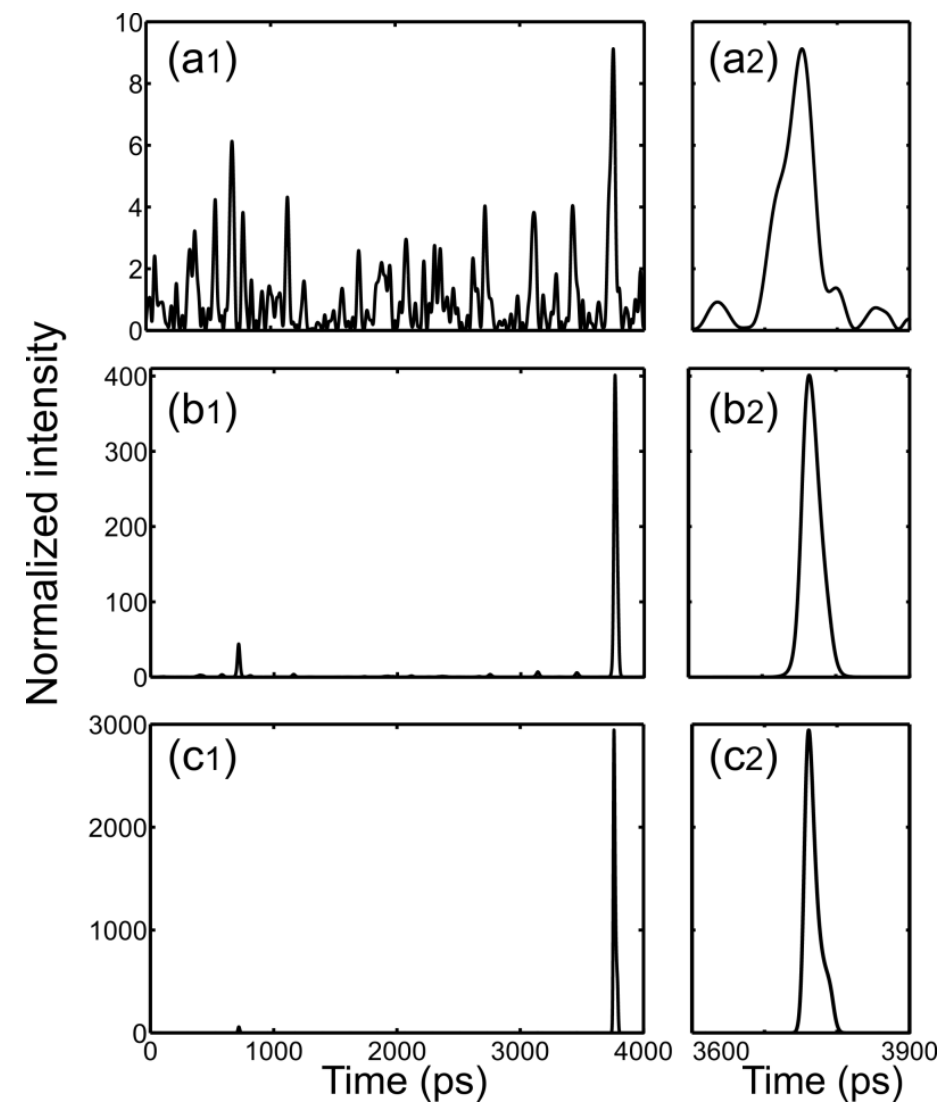

Figure 1 - Temporal intensity profiles of (a) the initial partially incoherent pump, (b) the signal and (c) the Stokes wave at the output of the Raman amplifier. Results are plotted on two different time scales: 4 ns (subplots 1) and 300 ps (subplots 2). Results are normalized with respect to the average power of the wave under study.

The existence of extreme events with non-negligible probability is also confirmed by the pdf of the amplified signal at different propagation lengths (results are calculated over a time window of $1.6 \mu \mathrm{s}$ ). Results are plotted in Figure 2(a) on a log-log scale and are compared with the pdf of the pump power (circles). Several important points can be outlined. First, these signal pdfs significantly differ from the pump one (analytically predicted by Eq. (3)) and are in clear agreement with a linear function when plotted on a log-log scale, consistent with the characteristic power law nature of extreme events. Such an algebraic decay is in agreement with the theoretical considerations developed in [26] or [28]. This algebraic long tail of the pdf reveals the existence of highly probable extreme events, which thus find their origin in the exponential and instantaneous nature of the Raman gain amplification. The exponent of the decay decreases with propagation distance, in qualitative agreement with the 1/z law proposed in [26]. A direct consequence is that the most extreme events have an increasing probability of appearance with 
the propagation distance. Note however that this pdf should be ultimately limited by the pdf of the pump [26]. This constitutes a major difference when compared to the gigantic amplification reported in counterpumped Raman amplifiers where transient effects may lead to amplified pulses having peaks powers that are orders of magnitude higher than the power of the pump beam [22].

Whereas powers more than ten times the average power have a probability of $4.10^{-5}$ for the pump, events with a power more than one hundred times the average power of the signal have a probability of occurrence above $7 \cdot 10^{-4}$. The pdf of the cascaded Stokes wave (Figure 2(b)) exhibits similar statistical characteristics so that extreme events can also appear around $1650 \mathrm{~nm}$, as already outlined in Figure 1.

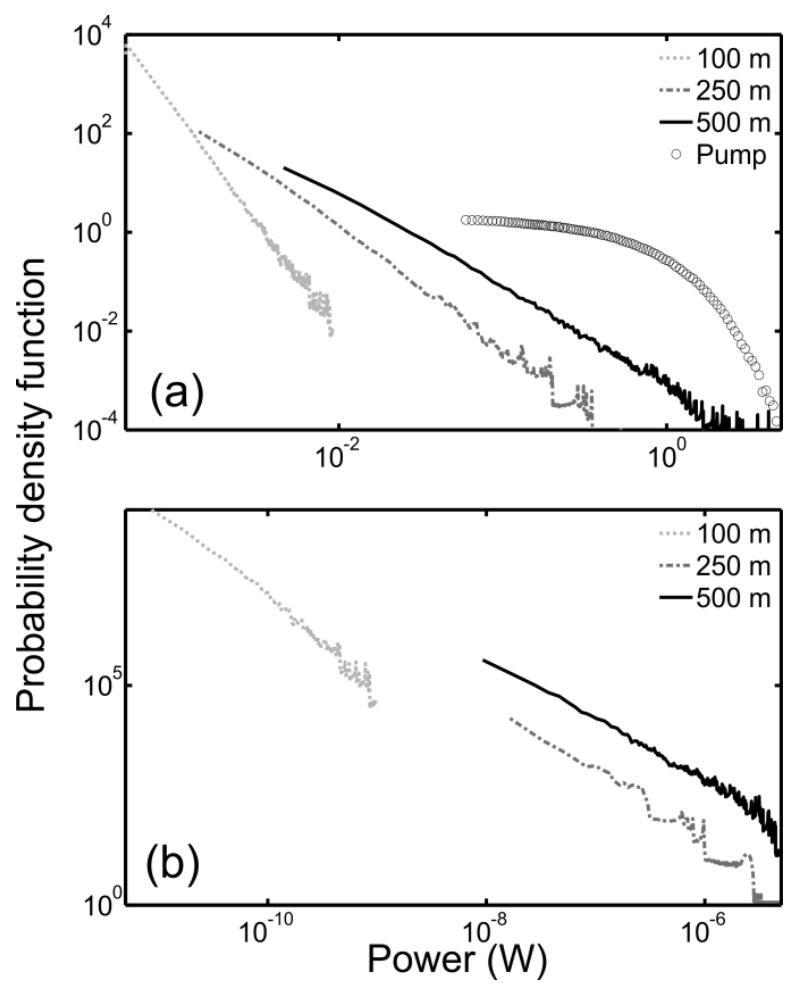

Figure 2 - Probability density function of the Signal (a) and the Stokes wave (b) plotted on a log-log scale. Results obtained at propagation lengths of $100 \mathrm{~m}, 250 \mathrm{~m}$ and $500 \mathrm{~m}$ (dotted light grey line, dashed grey line and solid black line respectively) are compared with the pdf of the pump (circles).

Though the pdf of the pump and signal can be experimentally recorded [24, 28], this technique unfortunately requires onerous dedicated devices. An indirect but more convenient mean to get an idea of the pump and signal temporal fluctuations is the normalized measure of the intensity autocorrelation function $\Gamma$ whose general definition reads [35] :

$$
\Gamma(\tau)=\frac{\lim _{T \rightarrow \infty} \frac{1}{T} \int_{-T / 2}^{T / 2} P(t+\tau) P(t) d t}{\lim _{T \rightarrow \infty} \frac{1}{T} \int_{-T / 2}^{T / 2} P^{2}(t) d t}
$$

The autocorrelation function is characterized by two quantities: the temporal width of the autocorrelation $\Sigma$ and the contrast ratio $\Upsilon$ of the autocorrelation. $\Sigma$ is the full width at half maximum of the function $\Gamma(\tau)-\lim _{\tau \rightarrow \infty} \Gamma(\tau) . \Upsilon$ is the ratio of the maximum value of $\Gamma$ over its minimum value, i.e 
$\Upsilon=\Gamma(0) / \lim _{\tau \rightarrow \infty} \Gamma(\tau)$. We recall that, for our pump field, which is characterized by Gaussian statistics, the variance of its power fluctuations equals two times its mean power, so that of $\Upsilon=2[33,35]$. Results are summarized in Figure 3 and outline the reshaping of this autocorrelation signal, starting from a continuous wave and evolving to a highly peaked structure along propagation in the fibre. Regarding the autocorrelation contrast $\Upsilon$, Figure 3(a-b) shows clearly that it is far from 1 (i.e., the contrast of a continuous wave). On the contrary, the contrast is very high and clearly different from the contrast 2 of the Gaussian pump, suggesting different statistical properties for the signal and pump waves. Such an increase in the contrast $\Upsilon$ well above the corresponding Gaussian value simply reflects the existence of highly probable extreme events, as discussed above through the analysis of the pdf. Figure 3(c) also shows that the contrast ratio of the cascaded Stokes wave is by more than one order of magnitude higher than the signal's one. The temporal width of the signal autocorrelation is close to the pump's one ( $35 \mathrm{ps,}$ Figure 3d). However, after a few hundreds of meters of propagation, temporal broadening occurs, which can be explained by the combined impact of the walk-off between the pump and the Stokes wave and a possible gain saturation [26]. We can also make out that the Stokes fluctuations are shorter than the pump or signal fluctuations.
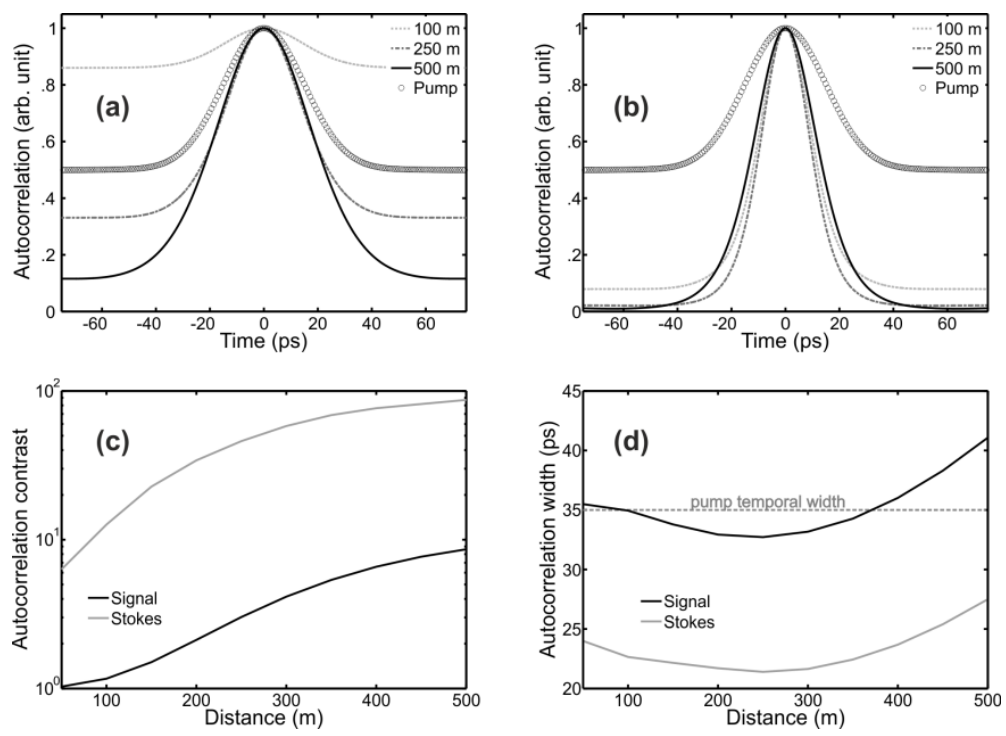

Figure 3 - Autocorrelation signal of the amplified signal (a) and the Stokes wave (b). Results obtained at propagation lengths of $100 \mathrm{~m}, 250 \mathrm{~m}$ and $500 \mathrm{~m}$ (dotted light grey line, dashed grey line and solid black line respectively) are compared with the autocorrelation of the pump (open circles). Longitudinal evolution of the autocorrelation contrast (c) and the autocorrelation width (d) for the signal amplified wave (solid black line) and the Stokes wave (solid grey line).

In order to further stress the link between the various waves, we have plotted on Figure 4 the crosscorrelations that can be computed between the pump intensity profile and the profile of the amplified signal or cascaded Stokes. Results show a pump-signal cross-correlation peak for which delay increases with propagation distance. Such a feature is linked to the walk-off between the incoherent pump and the continuous seed [25]: from the dispersive properties of the fibre, one can evaluate a walk-off coefficient $\delta_{\mathrm{SP}}=0.054 \mathrm{ps} . \mathrm{m}^{-1}$, which leads to an integrated walk-off that evolves linearly with the propagation distance (Figure 4(b), full black circles). The maximum delay remains comparable with the temporal width of the pump fluctuations so that transfer of intensity noise from the pump to the seed remains effective, as already predicted by a frequency analysis made by Fludger as early as 2001 [41]. 
Regarding the pump-cascaded Stokes cross-correlation, we can make out that the temporal position of the maximum of correlation does not follow such a simple rule: based on the pump-cascaded walk-off $\delta_{\mathrm{CP}}=0.15 \mathrm{ps} . \mathrm{m}^{-1}$ (see grey diamonds line) one cannot infer the result. Indeed, the situation is rather different: whereas the gain on the signal provided by pump acts during the whole propagation in the fibre with a similar strength, the exponential gain experienced by the cascaded Stokes wave is growing along propagation (due to the amplification of the signal that acts as a pump) and is maximum at the output of the amplifier. This explains that the signal and cascaded Stokes wave are close to present similar cross-correlation properties. In both cases, the cross-correlation peaks tend to broaden with propagation under the combined effect of walk-off and depletion. The pump-cascaded Stokes correlation peak is slightly broader than the pump-signal one.
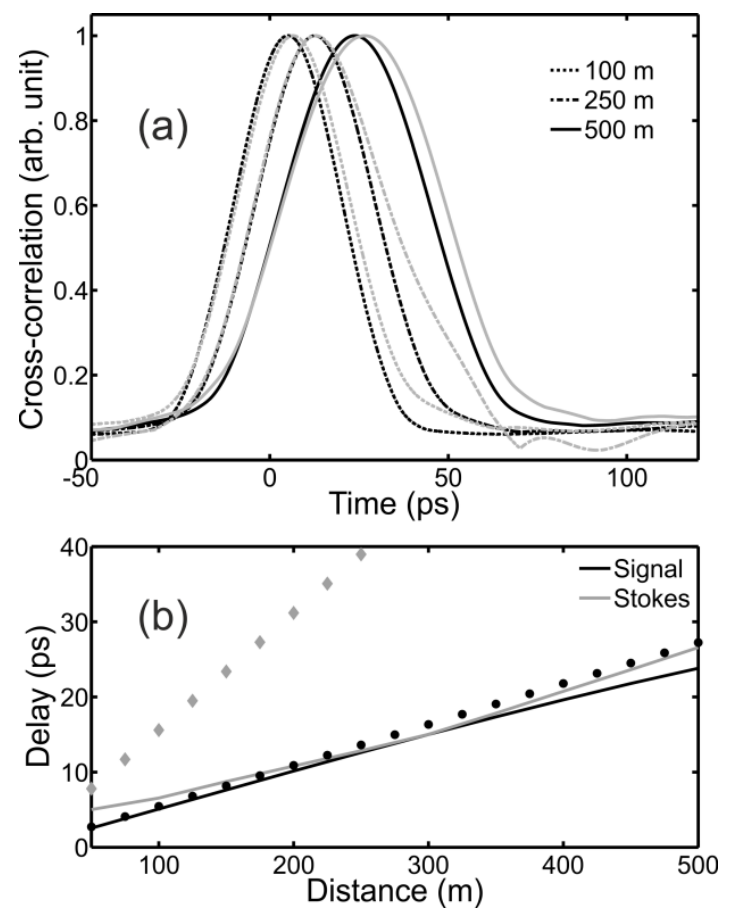

Figure 4 - (a) Cross-correlations between the pump and the signal (black line) and pump and Stokes wave (grey line). Results are normalized by the maximum value of the cross-correlation. Results obtained at propagation lengths of $100 \mathrm{~m}, 250 \mathrm{~m}$ and 500 $\mathrm{m}$ (dotted, dashed and solid lines respectively) are compared. (b) Evolution of the temporal delay of the cross-correlation-signal according to the distance of propagation for the signal and cascaded Stokes (solid black and grey lines respectively). Results are compared with the integrated walk-off (black circles and grey diamonds respectively).

Figure 5 shows the signal or cascaded Stokes intensity according to the pump intensity for a delay corresponding to the maximum correlation found in Figure 4. Given the analytical results of [26], we got more precisely interested in the logarithm of the intensity of the signal or the Stokes for different distances of propagation. For short distances, the relation between the two quantities is close to being strictly linear. This fully confirms the exponential amplification undergone by the signal: for small propagation distances, the output signal intensity can be known without ambiguity from the pump intensity profile delayed by the integrated walk-off value (that remains in this case very low). This potentially paves the way for an a-posteriori correction of the pump noise as proposed in [42]. However for higher propagation distances and even if the overall shape of the scatter diagram remains linear, the results are much more spread as a consequence of the combination of a higher value of walk-off and gain 
saturation. For example, we can notice that signal power as high as $10 \mathrm{~mW}$ can be recorded even for a zero instantaneous pump. Correction of the amplified signal would be in this case much more complex. Similar conclusions can be drawn for the Stokes wave that presents an increased dispersion of the data plotted on the scatter diagram.
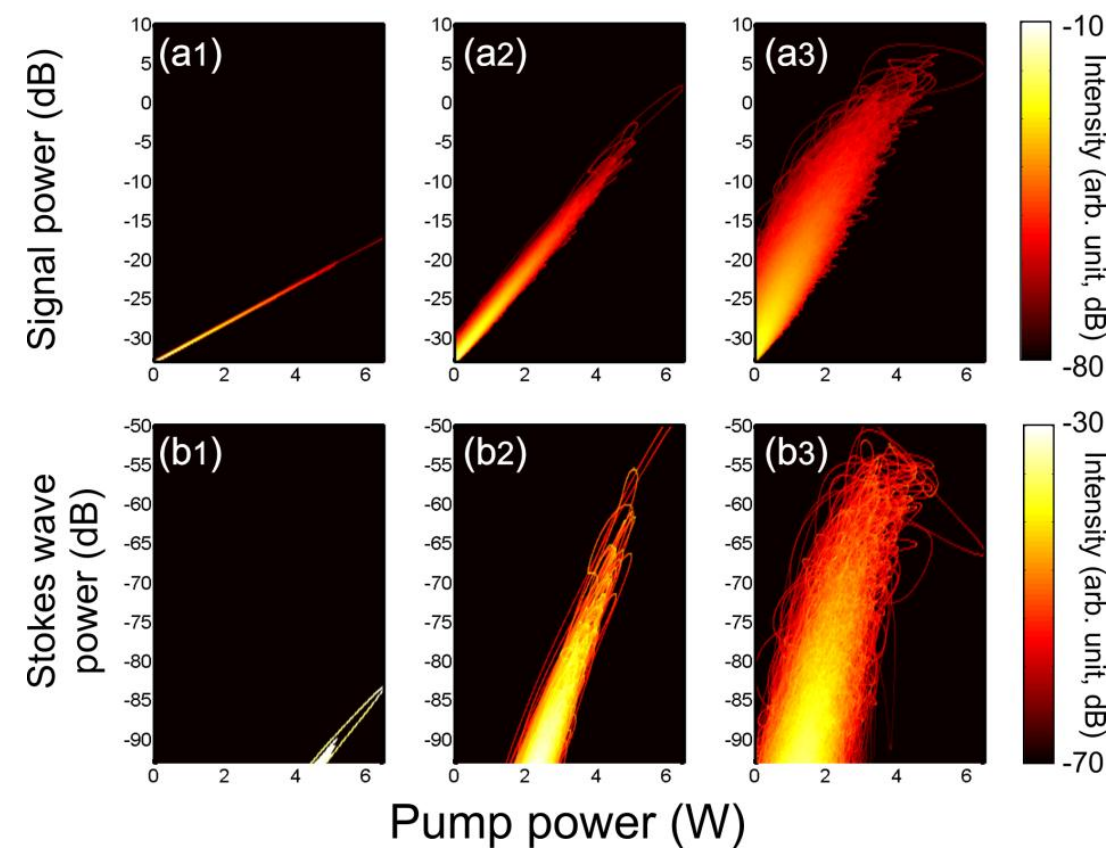

Figure 5 - Correlation diagrams between the pump and the amplified wave (a) and between the pump and the Stokes wave (b). Results are plotted at various distances of propagation $(100 \mathrm{~m}, 250 \mathrm{~m}$ and $500 \mathrm{~m}$, subplots 1,2 and 3 respectively). Note that the dynamics of color map is $70 \mathrm{~dB}$ for subplots 1 and only $40 \mathrm{~dB}$ for subplots 2 .

We finally got interested in the optical spectrum of the signal and the cascaded Stokes waves. Average spectra obtained at different distances of propagation are plotted on Figure 6. The input Dirac spectrum is significantly broadened and we can make out that the spectral extension of the spectra is much wider than what could be expected from the temporal duration of the spikes of light (around $25 \mathrm{ps,}$ leading to a Fourier limited spectrum of a few tens of GHz). Such a broadening is here explained by the cross-phase modulation of the intense pump on the continuous seed. In the context of passive propagation, an analytical treatment of the resulting optical spectrum has been proposed by Manassah [43]. Let us also remark that the process involved in the broadening occurring in a single pass element is different from the more complex turbulent-like dynamics that affects the spectral behaviour of Raman fibre cavity [16-18]. In the present study, spectral expansion is directly related to the initial pump fluctuations $[25,44]$ and therefore provides an efficient way to outline the fluctuations: a frequency offset optical bandpass spectral filtering is a practical solution to discriminate the most extreme spikes of light in the signal. Let us note that the spectral expansion of the Stokes wave is even larger and that for such a wave, a discrimination strategy based on a narrow frequency offset filter is not required: as outlined in Figure 1(c), the exponential gain of the cascading process is efficient enough to highlight the most extreme events. 


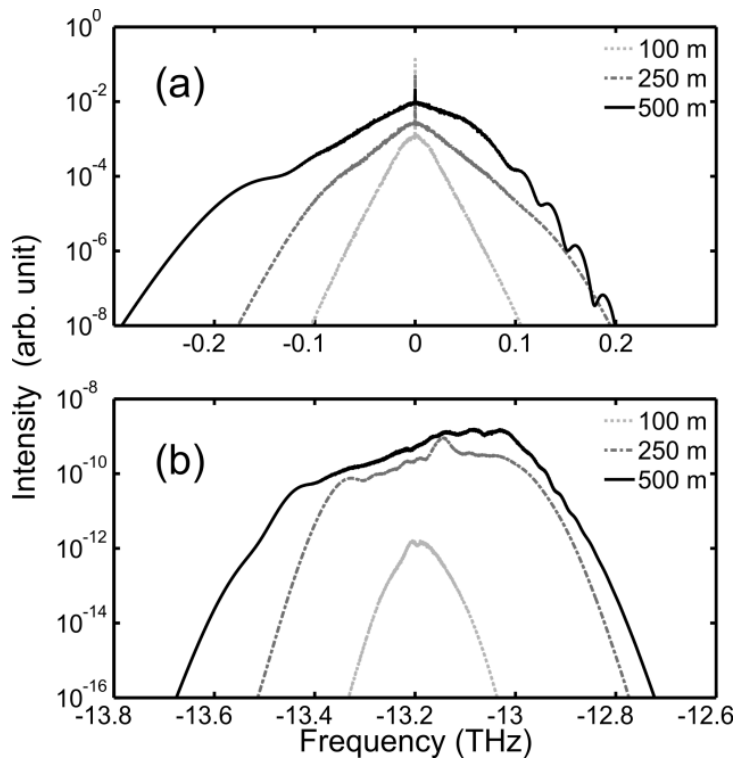

Figure 6 - Optical spectra of the amplified signal (a) and of the Stokes waves (b). Results obtained at propagation lengths of 100 $\mathrm{m}, 250 \mathrm{~m}$ and $500 \mathrm{~m}$ (light grey dotted, grey dashed and black solid lines respectively) are compared.

\section{Analysis of the evolution of a pulsed signal}

In this section, we now focus our attention on the evolution of an initial 40-GHz pulsed signal with a temporal width $(6.25 \mathrm{ps})$ below the coherence time of the pump. The goal is here to evaluate the impact of a co-propagating pumping scheme on a discrete Raman amplifier that may be used in the context of new telecommunications windows that cannot benefit from standard erbium doped fibre technologies [45, 46] or in the context of applications that require broad gain bandwidths [47]. Raman amplification can also be exploited to reinforce some nonlinear processings carried out in highly nonlinear elements [48-50].

In order to limit the consequences of pump depletion and gain saturation, we have used an initial peak-power of $5 \mathrm{~mW}$, i.e. an sub-mW input average power, which is lower than the one we typically consider in [25]. We have numerically considered the propagation of 64000 pulses that are then encoded by a pseudo random bit sequence. The optical modulator used for this purpose has a finite extinction ratio of $20 \mathrm{~dB}$.

The most intuitive and straightforward way to explore and evaluate signal variations at the output of the Raman amplifier is the observation of the optical eye-diagram as plotted in Figure 7 for selected distances in the amplifier. These eye-diagrams of the Return-to-Zero data stream clearly outline the very large fluctuations affecting both the 1 bits as well as the 0 bits. The poor fidelity of the amplified signal can be readily noticed even after $100 \mathrm{~m}$ of propagation. At the amplifier output, the fluctuations are striking and the fully closed eye indicates that the amplified pulse sequence cannot be suitable for transmission anymore. Once again, such large fluctuations have nothing to do with amplified spontaneous emission (ASE) or with multi-interference pattern. As the pulses remain well within their time slot, the fluctuations cannot be attributed to amplitude jitter induced by patterning effects such as intra channel four wave mixing [51]. The observed variations are definitively inherent to the exponential transfer of the intensity noise from the partially incoherent pump to the signal. 

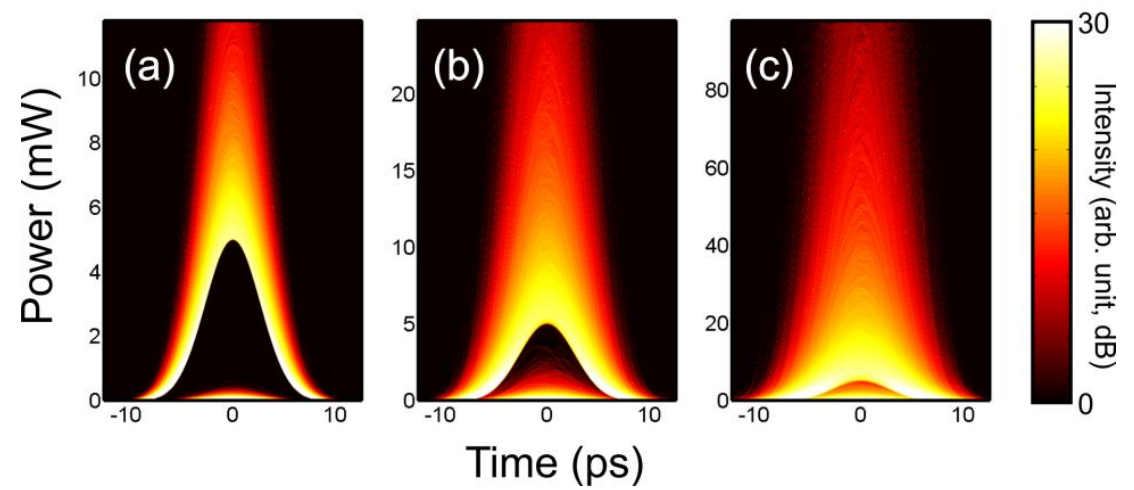

Figure 7- Optical eye-diagrams obtained at propagation lengths of (a) $100 \mathrm{~m}$, (b) $250 \mathrm{~m}$ and (c) $500 \mathrm{~m}$.

We have derived the resulting shot-to-shot statistics of the instantaneous optical power that can be recorded at the centre of the pulse, i.e. at the decision time (in our case, according to Figure $7, \mathrm{~T}=0$ ). Pdfs are summarized on Figure 8(a) both for 0's and 1's bits and for different distances of propagation. As expected, the results strongly deviate from a Gaussian behaviour and are close to an algebraic law [20, 25]. An important and direct consequence is that the assumption of Gaussian statistics required to link the widely-used Q-factor to the bit error rate are absolutely not fulfilled. In other words, in such a configuration, the standard deviation and average values are not sufficient to estimate accurately the level of errors. From our numerical simulations, we can make out that, at the output of the fibre amplifier, events with a peak power 20 times larger than the average power (i.e. above $1 \mathrm{~W}$ ) can be recorded with a non-negligible probability of $610^{-3}$. Consequently, significant detection errors may appear. In Figure 8(b), we have estimated the proportion of errors according to the optical decision threshold. Even for the optimum decision level, errors as frequent as one over one hundred are detected at the output of the amplifier, which outlines how prohibitive these fluctuations are. Such a proportion is not affected by the level of initial input power so that even for much lower average input powers, similar degradations are observed. With such a penalty, co-propagating pumping scheme has to be banned in Raman-enhanced optical regenerators [48-50]. As a direct consequence, it seems hard to combine the advantages of a bidirectional setup such as in $[52,53]$ with the benefits of Raman amplification. 

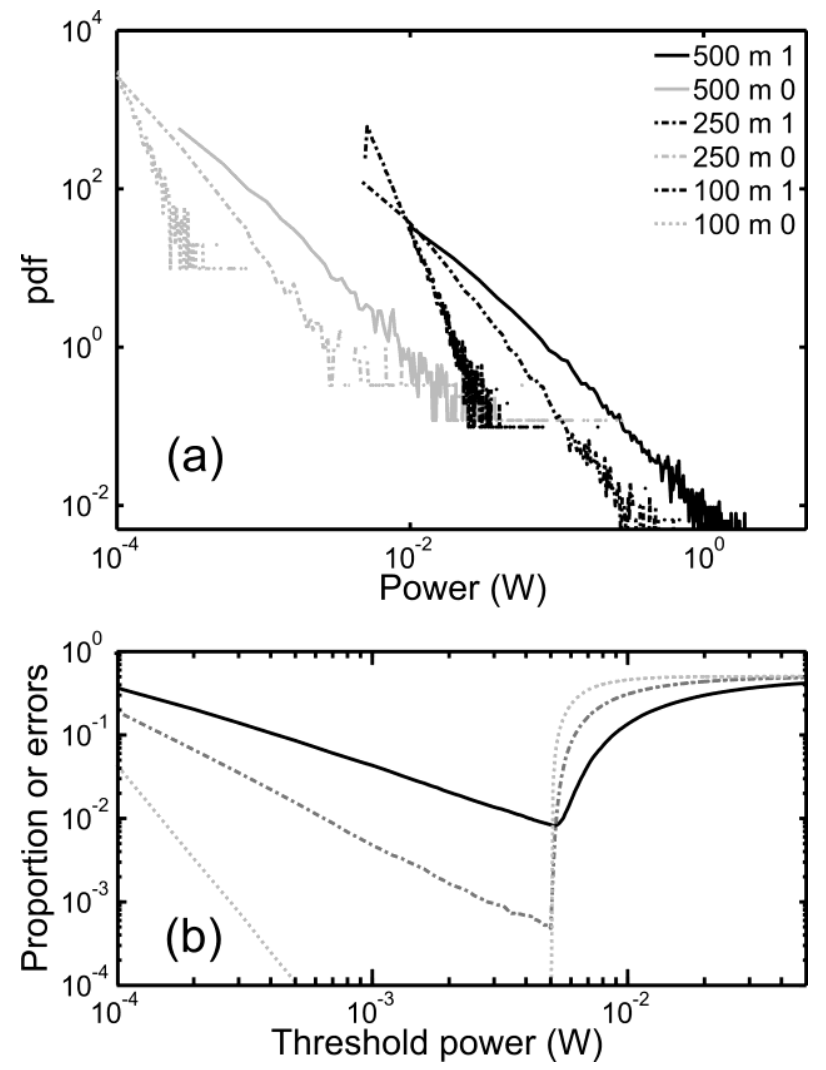

Figure 8- (a) Probability distribution function of the 1's and 0's peak power (black and grey lines respectively). Results obtained at propagation lengths of $100 \mathrm{~m}, 250 \mathrm{~m}$ and $500 \mathrm{~m}$ (dotted, dashed and solid lines respectively) are compared. (b) Evolution of the proportion of errors detected at the output of the amplifier according of the level of optical power threshold.

We have also investigated in more details the influence of the intensity noise transfer on the intensity autocorrelation signal. We consider here a purely periodic initial signal, i.e. without any pseudo random bit sequence. Interestingly, the level of the cross-correlation peak between a pulse and its neighbouring pulses can provide relevant information on the amplitude jitter degradation that affects the pulse train on a short time scale [54-56]. Results are summarized on Figure 9. The input pulse train exhibits a level of the central peak identical to the level of the cross-correlation peak. This level falls then drastically, which highlights the significant change of amplitude between two successive amplified pulses. Such a behaviour is in full agreement with the various experimental records based on the analysis of a $10 \mathrm{GHz}$ pulse picosecond train and detailed in [27]. Let us stress once again here that due to the breakdown of the Gaussian assumption, the quantitative estimates that can be derived from the analytics developed for example in [54] should be questioned. Using the temporal width of the cross-correlation peak, we can find that there is no significant increase of the timing jitter induced by the amplification process [54], confirming therefore that the main source of degradation is amplitude jitter. Results dealing with the Stokes wave (Figure 9(b)) show a very low level of correlation, indicating that extremely large shot-to-shot variations occur. 


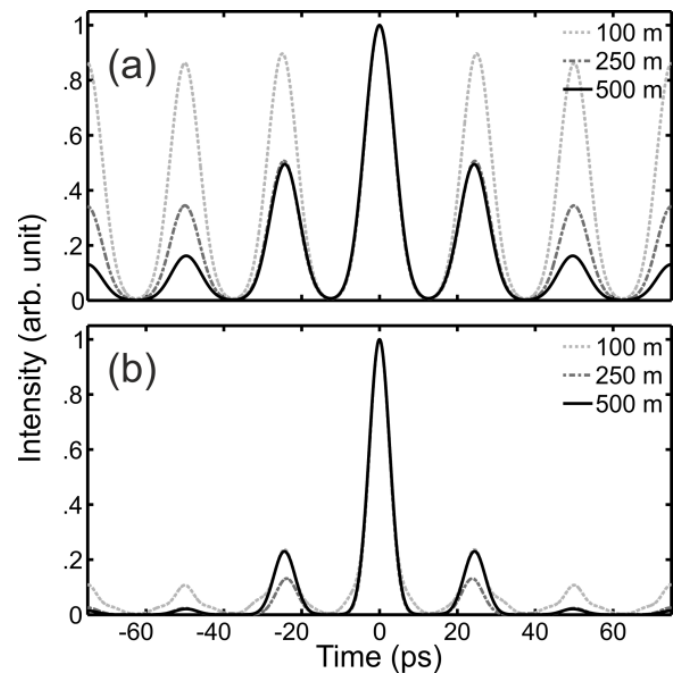

Figure 9 - Autocorrelation signals of the signal (a) and the Stokes wave (b) recorded at different distances of propagation. Results obtained at propagation lengths of $100 \mathrm{~m}, 250 \mathrm{~m}$ and $500 \mathrm{~m}$ (light grey dotted, grey dashed and black solid lines respectively) are compared.

We complement our temporal measurements by performing a spectral radio frequency (RF) analysis of the pulse train. Note that ideally, this data is linked to the autocorrelation signal we have previously described through the Wiener-Khinchin theorem. RF spectra are intensively used to characterize the optical amplifier properties: the measurement of the relative intensity noise (RIN) has indeed become a standard analysis to discuss the performance of optical amplifiers [57]. Other authors have also proposed to extract from the comb-like RF spectrum the amplitude and timing jitters of the periodic signal [58]. However, this latest application is not relevant to the present work as it requires the analysis of a large number of harmonics peaks and as it is based on the strong assumption of small variations having a Gaussian probability distribution, which is clearly not our case. Closer to the context of our study, other works have taken benefit of the degraded RF spectrum and the dependence of the RIN transfer according to walk-off values to evaluate the fluctuations properties of a Raman fibre laser [34].

Our results are summarized on Figure 10(a) and clearly exhibit the increasing level of noise contained around the $40 \mathrm{GHz}$ periodic sidebands. Let us note however that compared to the experimental results described in [27], no additional peaks in the vicinity of the $40 \mathrm{GHz}$ components is observed. Such experimental peaks were linked to the complex modal nature of the emission of the Raman fibre cavity under use $[59,60]$ which deviates from the simplified assumptions made for modelling the pump wave (our Gaussian optical spectrum with a random phase can be experimentally realized by spectral filtering of an ASE source [19, 24, 28]). From the RF measurements, no spectral broadening of the comb structure is visible, which confirms that the duration of the pulses after amplification does not radically differ from the input pulse stream. 

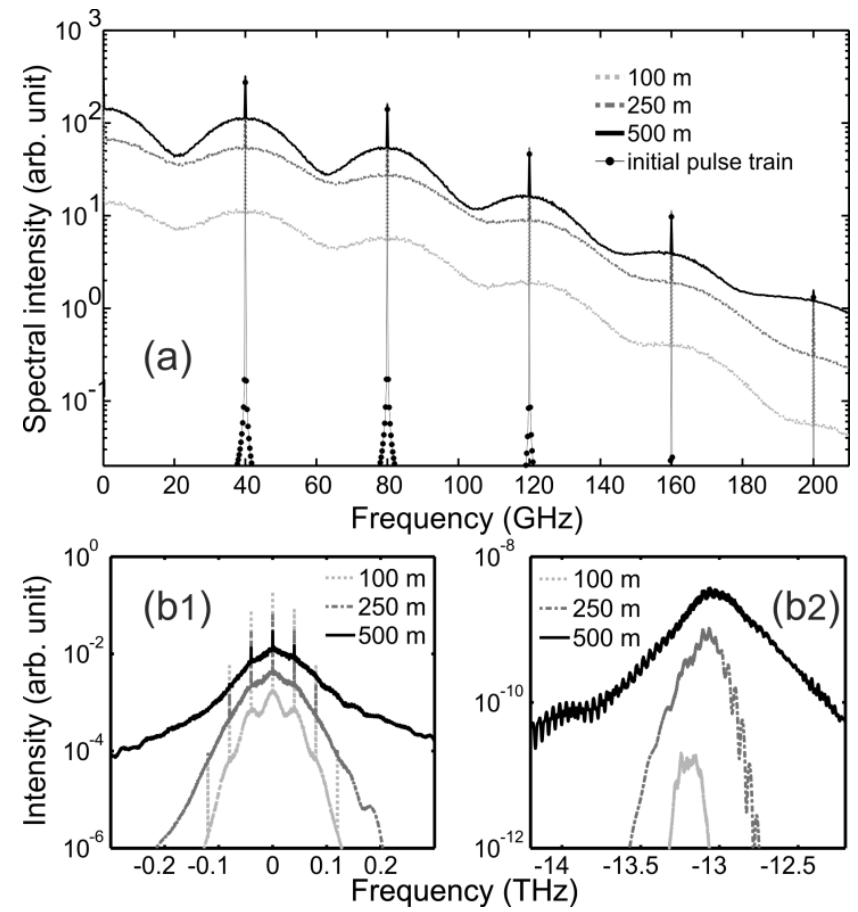

Figure 10 - (a) Radio Frequency spectrum (arb. units, results normalized with respect to the average power) and optical spectrum of the signal (b1) and cascaded Stokes wave (b2). Results are compared to various propagation lengths of $100 \mathrm{~m}, 250$ $\mathrm{m}$ and $500 \mathrm{~m}$ (light grey dotted, grey dashed and black solid lines respectively). RF spectrum of the input pulse train is plotted with black circles).

The optical spectrum (Figure 10b) confirms the dramatic drop of the optical signal to noise ratio (as usually measured in the telecommunication context [57]), in agreement with the experimental records detailed in [27]. The optical spectra of the cascaded Stokes wave do not exhibit clear $40 \mathrm{GHz}$ components, which is consistent with the results of Figure 9(b) that have outlined the low correlation between structures delayed of 25 ps. Compared to the RF measurements, we can make out that the expansion of the optical spectrum of the signal is much more pronounced. This suggests that the phase of the pulses is also heavily altered during the amplification process.

This is why it is also of interest to wonder if the serious degradations experienced on the intensity profile are reproduced on the phase profile. We have therefore evaluated pdfs of the optical signal phase when measured at the centre of the pulse. Results are summarized on Figure 11 and clearly outline the phase dithering that occurs during amplification: whereas the input signal is here made of pulses having all the same phase, the output pulse train exhibits a distribution of the phase that is widely spread between $-\pi$ and $\pi$ and that tends to approach a uniform distribution between these two values. These results stress that such lumped Raman amplifier operated in a copropagating pumping scheme are absolutely not suitable for amplification of phase encoded data stream. It is worth noting that the same statistics carried out on the 0's bit of information (i.e. pulses attenuated by $20 \mathrm{~dB}$ due to the finite extinction ratio of the modulator) shows a similar spreading of the statistical distribution. Such an observation suggests that selfphase modulation undergone by the pulse train is not the main component of this degradation (which differs from the conclusions of [25] where input pulses were much more powerful). 


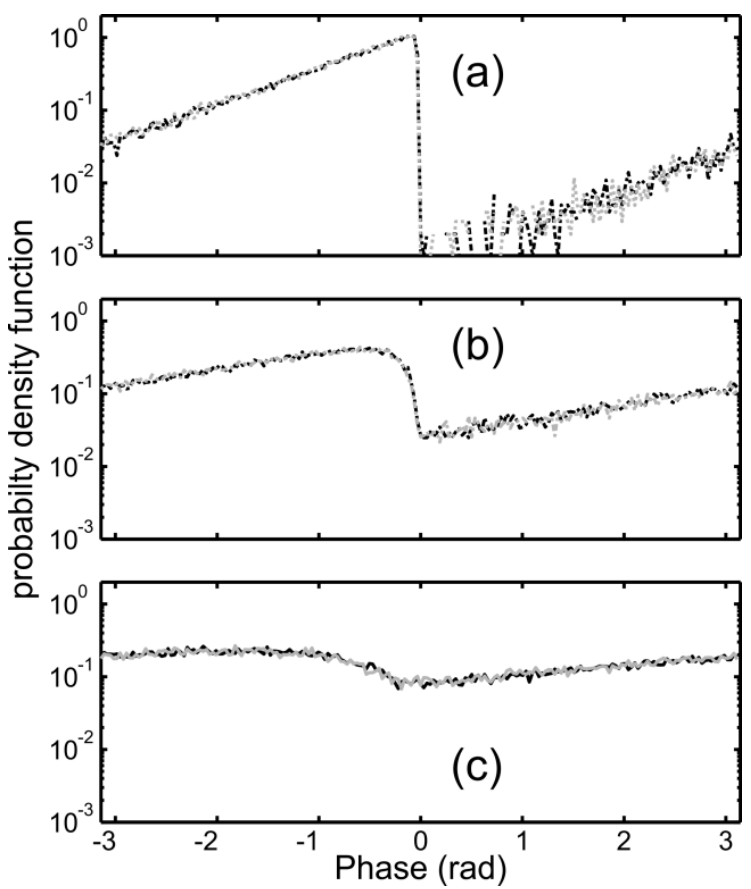

Figure 11 - Pdfs of the 1's and 0's phase (black and grey lines respectively) for different propagation lengths: $100 \mathrm{~m}$ (a), $250 \mathrm{~m}$ (b) and $500 \mathrm{~m} \mathrm{(c).}$

One question that arises is the correlation of those phase degradations with the previously described intensity fluctuations. The constellation diagram provides an efficient way to visualize simultaneously the intensity and phase degradations. Such a diagram obtained at the output of the amplifier is plotted on Figure 12 for two scales of visualization. It points out that the fluctuations are not randomly distributed in the IQ space: the data clearly distribute along a multi-turn spiral. The nonlinear dynamics is therefore different to the one recently reported in [61] and interpreted as random walks and Levy flights in supercontinuums. 

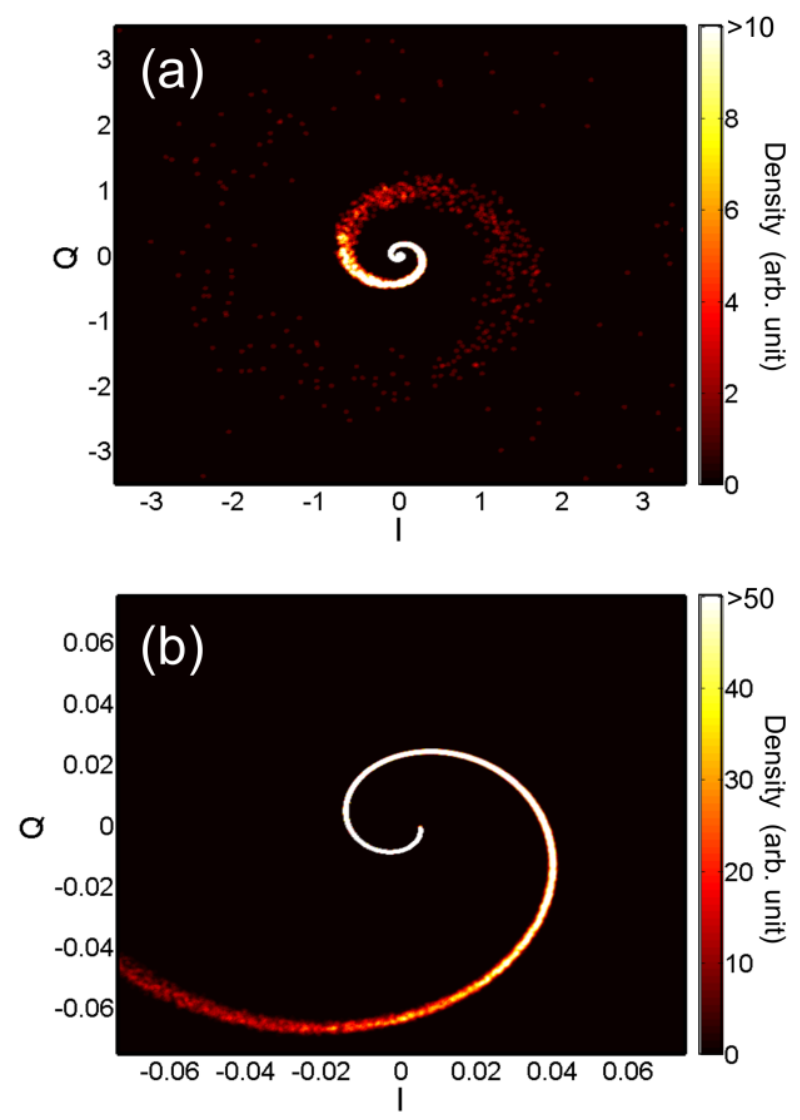

Figure 12- IQ Diagrams of the output pulse train plotted on two different scales. (b) is a magnification of the central part of (a).

In order to confirm this observation, we have plotted in Figure 13(a) the phase at the instant of detection according to the logarithm of the peak power of the signal pulses. The corresponding scatter plot outlines the strong connection between the phase of the signal and its peak-power. Indeed, as we have already discussed, peak power of the signal pulse is related to the pump fluctuation through an exponential law and the phase is also linked to the pump via the cross-phase modulation through a linear law. Remark that for higher peak powers, the correlation between phase and peak power decreases. Figure 13(a) also confirms the wrapping of the phase: the apparent uniform distribution of Figure 11 is to be attributed to the $2 \pi$ phase ambiguity that leads to an overlap of various parts of a broadened and tailed distribution. However, the wrapping ambiguity should be overcome using the aforementioned correlation between phase and peak-power. It is indeed possible to reconstruct the phase and the associated pdf without the ambiguity, as presented in Figure 13(b). We can then notice that phase shifts as high as 20 rad can be experienced and interestingly, the pdf when plotted on a semi-logarithmic exhibits a linear trend consistent with the linear law linking the pump intensity and the phase induced by cross-phase modulation. 

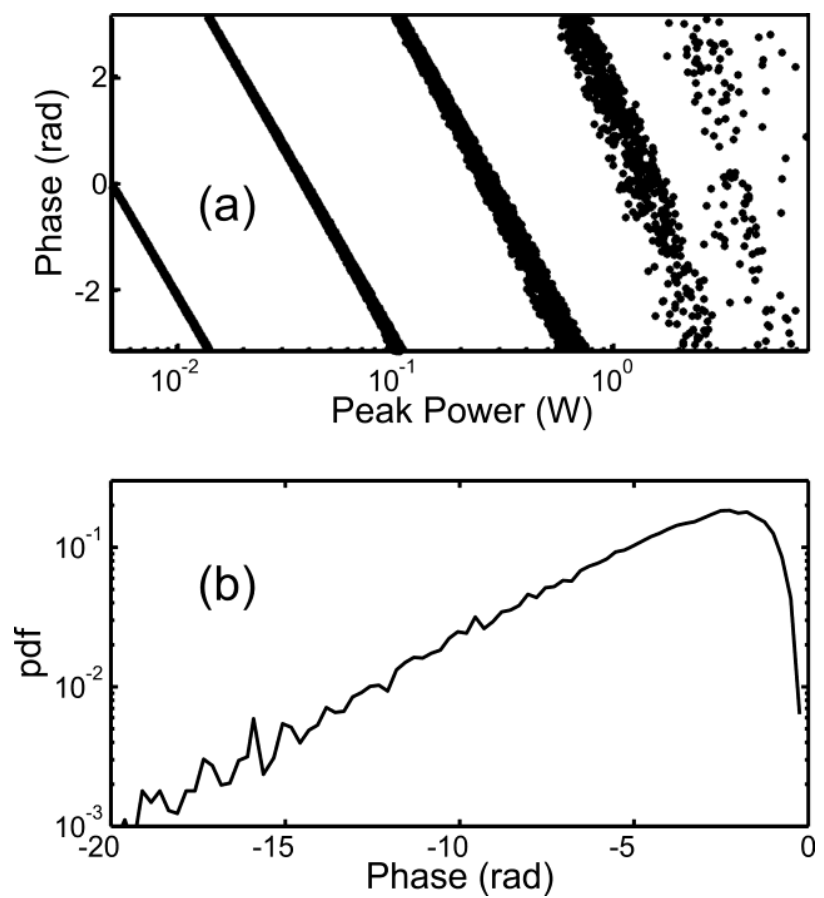

Figure 13- (a) Correlation of the phase and the peak power of the 1's of the amplified signal. (b) Pdf of the phase after correction of the wrapping ambiguity

\section{Conclusion}

Based on a set of numerical simulations of the amplification of continuous and pulsed signals, we have presented in this paper an overview of the generation of extreme events in a lumped fibre amplifier pumped by a partially incoherent wave in a co-propagating scheme with a low walk-off value. We have provided some clues for a better understanding of the emergence of rare but intense spikes of light. We have also highlighted the impact on the phase and spectrum of the amplified signal and investigated the generation and properties of the cascaded Stokes wave.

The numerical results presented here have confirmed several experimental observations detailed in ref [27] where a set of unconventional methods were used to qualitatively stress the highly damaging impairments of these pulse-to-pulse fluctuations. The very large range of intensity and phase fluctuations prevents the use of the highly nonlinear discrete Raman amplifier pumped in a co-propagating scheme for telecommunication applications. However, the ability of such devices to provide extreme statistics for the intensity or very large fluctuations of the phase may find interesting applications in other fields of optics where signals with non-Gaussian statistics are required.

\section{Acknowledgement}

We would like to thank Guy Millot, Antonio Picozzi, Bertrand Kibler, John Dudley and Julien Fatome for fruitful and stimulating discussions. This research was supported by the Agence Nationale de la Recherche (ANR MANUREVA project: ANR-08-SYSC-019 and ANR OPTIROC), by the Labex ACTION and by the Conseil Régional de Bourgogne (PhotCom Pari scheme). 


\section{References}

[1]. Solli D R et al., 2007, Optical rogue waves. Nature. 450: p. 1054.

[2]. Dudley J M et al., 2010, Extreme events in optics: Challenges of the MANUREVA project. Eur. Phys. J. Special Topics. 185(1): p. 125-133.

[3]. Hammani K et al., 2012, Nonlinear spectral shaping and optical rogue events in fiber-based systems. Opt. Fiber. Technol. 18(5): p. 248-256.

[4]. Taki M et al., 2010, Third-order dispersion for generating optical rogue solitons. Phys. Lett. A. 374(4): p. 691-695.

[5]. Hammani K et al., 2010, Emergence of rogue waves from optical turbulence. Phys. Lett. A. 374(34): p. 3585-3589.

[6]. Genty G et al., 2010, Collisions and turbulence in optical rogue wave formation. Phys. Lett. A. 374(7): p. 989-996.

[7]. Hasegawa A and Tappert F, 1973, Transmission of stationary nonlinear optical pulses in dispersive dielectric fibers. I. Anomalous dispersion. Appl. Phys. Lett. 23(3): p. 142-144.

[8]. Skryabin D V and Gorbach A V, 2010, Looking at a soliton through the prism of optical supercontinuum. Rev. Modern Physics. 82: p. 1287-1299.

[9]. Akhmediev N N and Korneev V I, 1986, Modulation instability and periodic-solutions of the nonlinear Schrödinger equation. Theor. Math. Phys. 69(2): p. 1089-1093.

[10]. Dudley J M et al., 2009, Modulation instability, Akhmediev Breathers and continuous wave supercontinuum generation. Opt. Express. 17(24): p. 21497-21508.

[11]. Hammani K et al., 2011, Peregrine soliton generation and breakup in standard telecommunications fiber. Opt. Lett. 36(2): p. 112-114.

[12]. Kibler B et al., 2011, Rogue waves, rational solitons and wave turbulence theory. Phys. Lett. A. 375(35): p. 3149-3155.

[13]. Peleg A, 2004, Log-normal distribution of pulse amplitudes due to Raman cross talk in wavelength division multiplexing soliton transmission. Opt. Lett. 29(17): p. 1980-1982.

[14]. Chung Y J and Peleg A, 2005, Strongly non-Gaussian statistics of optical soliton parameters due to collisions in the presence of delayed Raman response. Nonlinearity. 18(4): p. 1555-1574.

[15]. Vergeles S and Turitsyn S K, 2011, Optical rogue waves in telecommunication data streams. Phys. Rev. A. 83(6): p. 061801.

[16]. Turitsyna E G et al., 2009, Optical turbulence and spectral condensate in long-fiber lasers. Phys. Rev. A. 80: p. 031804.

[17]. Churkin D V et al., 2011, Extreme value statistics in Raman fiber lasers. Opt. Lett. 18: p. 3617-3619.

[18]. Randoux S and Suret P, 2012, Experimental evidence of extreme value statistics in Raman fiber lasers. Opt. Lett. 37(4): p. 500-502.

[19]. Hammani K et al., 2009, Emergence of extreme events in fiber-based parametric processes driven by a partially incoherent wave. Opt. Lett. 34(8): p. 1138-1140.

[20]. Hammani K et al., 2008, Optical rogue-wave fluctuations in fiber Raman amplifiers. Opt. Express. 16(21): p. $16467-16474$.

[21]. Borlaug D et al., 2008, Extreme value statistics in silicon photonics. IEEE Photon. J. 1(1): p. 33-39.

[22]. Kozlov V V et al., 2011, Exact solution for the gigantic amplification of ultrashort pulses in counterpumped Raman amplifiers. Opt. Lett. 36(9): p. 1632-1634.

[23]. Hammani K et al., 2009, Soliton generation in a microstructured fiber by fourth order scalar modulation instability. IEEE Photon. J. 1(3): p. 205-212.

[24]. $\mathrm{Xu} \mathrm{Y} \mathrm{and} \mathrm{Murdoch} \mathrm{S} \mathrm{G,} \mathrm{2010,} \mathrm{Gain} \mathrm{statistics} \mathrm{of} \mathrm{a} \mathrm{fiber} \mathrm{optical} \mathrm{parametric} \mathrm{amplifier} \mathrm{with} \mathrm{a} \mathrm{temporally}$ incoherent pump. Opt. Lett. 35(6): p. 626-628.

[25]. Finot $\mathrm{C}$ et al., 2009, Selection of extreme events generated in Raman fiber amplifiers through spectral offset filtering. IEEE J. Quantum Electron. 46(2): p. 205-213.

[26]. Hammani $\mathrm{K}$ et al., 2011, Extreme statistics in Raman fiber amplifier : from analytical description to experiments. Opt. Commun. 284: p. 2594-2603.

[27]. Hammani K and Finot C, 2012, Experimental signatures of extreme optical fluctuations in lumped Raman fiber amplifiers. Opt. Fiber. Technol. 18(2): p. 93-100. 
[28]. Xu Y Q and Murdoch S G. 2010. Gain statistics of a fiber optical Raman amplifier with a temporally incoherent pump. in Optical Communication (ECOC), 2010 36th European Conference and Exhibition on. 2010.

[29]. Agrawal G P. 2001 Applications of nonlinear fiber optics, San Francisco, CA: Academic Press.

[30]. Bromage J, 2004, Raman amplification for fiber communications systems. J. Lightw. Technol. 22(1): p. 7993.

[31]. Headley C and Agrawal G P. 2005 Raman amplification in fiber optical communications: Academic Press.

[32]. Betlej A et al., 2005, Increased Stokes pulse energy variation from amplified classical noise in a fiber Raman generator. Opt. Express. 13(8): p. 2948-2960.

[33]. Vanholsbeeck F et al., 2005, The role of pump incoherence in continuous-wave supercontinuum generation. Opt. Express. 13(17): p. 6615-6625.

[34]. Schröder J and Coen S, 2009, Observation of high contrast, fast intensity noise of a continuous wave Raman fiber laser. Opt. Express. 17(19): p. 1644-1649.

[35]. Goodman J W. 1985 Statistical optics: John Wileay and Sons.

[36]. Garcia L et al., 2002, Influence of classical pump noise on long-pulse multiorder stimulated Raman scattering in optical fiber. J. Opt. Soc. Am. B. 19(11): p. 2727-2736.

[37]. Landahl E et al., 1998, A simple analytic model for noise shaping by an optical fiber Raman generator. Opt. Commun. 150: p. 339-347.

[38]. Aalto A et al., 2010, Extreme-value statistics in supercontinuum generation by cascaded stimulated Raman scattering. Opt. Express. 18(2): p. 1234-1239.

[39]. Dahan D and Eisenstein G, 2004, The properties of amplified spontaneous emission noise in saturated fiber Raman amplifiers operating with CW signals. Opt. Commun. 236: p. 279-288.

[40]. Headley C and Agrawal G P, 1995, Noise characteristics and statistics of picosecond Stokes pulses generated in optical fibers through stimulated Raman scattering. IEEE J. Quantum Electron. 31(11): p. 2058-2067.

[41]. Fludger C et al., 2001, Pump to signal RIN transfer in Raman fiber amplifiers. J. Lightw. Technol. 19(8): p. 1140-1148.

[42]. Ataie V et al., 2012, Pump noise cancellation in parametric wavelength converters. Opt. Express. 20(26): p. B71-B76.

[43]. Manassah J T, 1991, Induced phase modulation by noisy fields. Opt. Lett. 16(18): p. 1379-1381.

[44]. Ravet G et al. 2007. Spectral broadening in Raman fiber amplifier pumped by partially coherent wave. in CLEO Europe. 2007. Munich.

[45]. Islam M N, 2002, Raman amplifiers for telecommunications. IEEE J. Sel. Top. Quantum Electron. 8(3): p. 548-559.

[46]. Mori A et al., 2003, Ultra-wide-band tellurite-based fiber Raman amplifier. J. Lightw. Technol. 21(5): p. 1300-1306.

[47]. Miyamoto $\mathrm{T}$ et al., 2005, Highly nonlinear fiber-based lumped fiber Raman amplifier for CWDM transmission systems. J. Lightw. Technol. 23(11): p. 3475-3483.

[48]. Boscolo S et al., 2006, Design of Raman-based Nonlinear Loop Mirror for all-optical 2R regeneration of Differential -Phase-Shift-Keying transmission. IEEE J. Quantum Electron. 42(7): p. 619-624.

[49]. Finot $\mathrm{C}$ et al., 2011, Active Mamyshev regenerator. Optical Review. 18(3): p. 257-263.

[50]. Wang W et al., 2005, Raman-enhanced regenerative ultrafast all-optical fiber XPM wavelength converter. J. Lightw. Technol. 23(3): p. 1105-1115.

[51]. Essiambre R J et al., 1999, Intra-channel cross-phase modulation and four-wave mixing in high-speed TDM systems. Electron. Lett. 35(18): p. 1576-1578.

[52]. Provost L et al., 2008, Analysis of a two-channel 2R all-optical regenerator based on a counter-propagating configuration. Opt. Express. 16(3): p. 2264-2275.

[53]. Matsumoto M, 2006, Efficient all-optical 2R regeneration using self-phase modulation in bidirectional fiber configuration. Opt. Express. 14(23): p. 11018-11023.

[54]. Fatome $\mathrm{J}$ et al., 2008, All-optical measurements of background, amplitude, and timing jitters for high speed pulse trains or PRBS sequences using autocorrelation function. Opt. Fiber. Technol. 14: p. 84-91.

[55]. Vo T D et al., 2010, Simultaneous multi-impairment monitoring of $640 \mathrm{~Gb} / \mathrm{s}$ signals using photonic chip based RF spectrum analyzer. Opt. Express. 18(4): p. 3938-3945.

[56]. Dinu M et al., 2006, Optical performance monitoring using data stream intensity autocorrelation. J. Lightw. Technol. 24(3): p. 1194-1202. 
[57]. Baney D M et al., 2000, Theory and measurement techniques for the noise figure of optical amplifiers. Opt. Fiber. Technol. 6: p. 122-154.

[58]. Von der Linde D, 1986, Characterization of the noise in continuously operating mode-locked lasers. Appl. Phys. B. 39(4): p. 201-217.

[59]. Babin S A et al., 2007, Experimental demonstration of mode structure in ultralong Raman fiber lasers. Opt. Lett. 32(9): p. 1135-1137.

[60]. Babin S A et al., 2005, Relative intensity noise in cascaded-Raman fiber lasers. IEEE Photon. Technol. Lett. 17(12): p. 2553-2555.

[61]. Wetzel B et al., 2012, Random walks and random numbers from supercontinuum generation. Opt. Express. 20(10): p. 11143-11152. 